\title{
Experiment on the Shielding by Hollow Conducting Tubes
}

\author{
Fethi Choubani, Jacques David, Member, IEEE, and Nikos E. Mastorakis, Senior Member, IEEE
}

\begin{abstract}
This paper describes several practical aspects of shielding provided by openings such as ventilation vents acting as waveguides. Fundamental properties of electromagnetic waves in the interior of a hollow conducting cylindrical tube are presented. Then the guidelines for oscillator's design and harmonic generation by nonlinearities are presented. A consequent wideband setup having low cost and common devices as well as high dynamic range is proposed to perform reliable measurements from $50 \mathrm{MHz}$ to 1.5 GHz. Measurements of circular cylindrical waveguide effectiveness point out the simplicity of the systematic procedure and illustrate the effects of apertures on shielding walls. Shielding loss achieved by these apertures at frequencies below cutoff allows engineers to gain more insight into analysis and design of shields with intentional discontinuities used to exclude and to confine electromagnetic interference.
\end{abstract}

Index Terms-Apertures, cutoff frequencies, interferences, oscillators, shielding walls, waveguides.

\section{INTRODUCTION}

$\mathbf{M}$ ODERN electronic systems incorporate a large number of interconnected devices, cohabiting in the same electromagnetic environment. The latter, when sufficiently polluted with undesired electromagnetic radiations, interferes with the operation of many electronics systems.

Electromagnetic shielding has been one of the most radical ways to deal with these interferences, hence assuring electromagnetic compatibility (EMC) [1], [2]. Nevertheless, shielding problems still remain difficult to handle because intentional discontinuities in shielding walls, such as ventilation holes, might violate shielding integrity.

The intent of this paper is to clarify the behavior of electromagnetic fields in waveguides below cutoff frequencies, so to explain shielding effectiveness provided by different kinds of holes in a conducting panel.

A waveguide transmission experiment is implemented easily using available equipment in most microwaves laboratories, such as common spectrum analyzers and guiding structures.

In addition to this infrastructure, free and quartz-based oscillators loaded with nonlinear elements are built to generate harmonics up to $2 \mathrm{GHz}$.

The main objective of this work is to develop a systematic shielding measurements procedure that

Manuscript received May 12, 2004; revised September 14, 2005.

F. Choubani is with the University of 7 th of November at Carthage, Ecole Superieure des Communications de Tunis, Tunis, Tunisia (e-mail: fethi. choubani@supcom.rnu.tn).

J. David is with ENSEEIHT, National Polytechnic Institute of Toulouse, Toulouse, France (e-mail: jdavid@enseeiht.fr).

N. E. Mastorakis is with MIUE, Hellenic Naval Academy, Greece (e-mail: mastor@ieee.org).

Digital Object Identifier 10.1109/TEMC.2006.873872

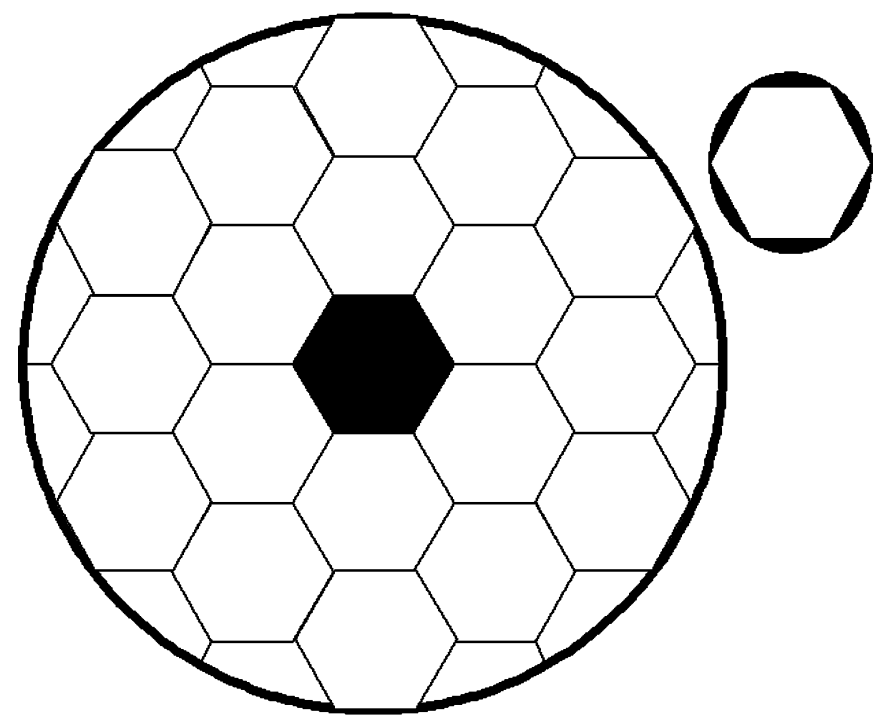

Fig. 1. Waveguide honeycomb vents.

1) is simple, repeatable, and accurate enough;

2) needs low-cost equipment and no sample preparation;

3) covers large frequency band and has high dynamic range.

\section{WAVEGUIDES BELOW CUTOFF}

A panel assembly of honeycomb-shaped tubes is shown in Fig. 1. Its apertures can be modeled as simple geometrical shapes such as rectangular slots or circular holes forming the whole frame. Each cell acts as a waveguide.

Therefore, to study the electromagnetic field penetration through these apertures, waveguide principles shall be addressed.

All waveguides have many properties in common [3], [4]. In this paper, we shall be concerned with fundamental properties of electromagnetic waves in the interior of uniform cylindrical waveguides.

The wave propagation in guiding structures is characterized by varying electromagnetic fields subjected to appropriate boundary conditions, such as the tangential component of the electric field and the normal component of the magnetic field vanishing at the perfect metal surface. These fields may satisfy the boundary conditions in a number of ways called modes, which are broadly divided into two classes:

1) transverse Electric (TE or $\mathrm{H})$ modes, the electric field being transverse to the direction of propagation;

2) transverse Magnetic (TM or E) modes, the magnetic field being transverse to the direction of propagation. 
The lowest mode having the lowest frequency and longest wavelength of propagation is called the dominant mode.

For each mode, electromagnetic fields are generally expressed in an orthogonal curvilinear cylindrical coordinate system $\left(u_{r}, u_{\theta}, z\right)$ as follows:

$$
\left\{\begin{array}{l}
E(r, \theta, z, t)=E(r, \theta) \exp (-\gamma z) \cdot \exp (j \omega t) \\
H(r, \theta, z, t)=H(r, \theta) \exp (-\gamma z) \cdot \exp (j \omega t)
\end{array}\right.
$$

where

$$
\begin{array}{ll}
\gamma=\left(2 \pi / \lambda_{\mathrm{g}}\right)=\sqrt{k_{\mathrm{c}}^{2}-k_{0}^{2}} & \text { propagation constant; } \\
k_{0}=\omega \sqrt{\mu_{0} \varepsilon_{0}}=\left(2 \pi / \lambda_{0}\right) & \text { free space propagation con- } \\
& \text { stant; } \\
k_{\mathrm{c}}=\left(2 \pi / \lambda_{\mathrm{c}}\right) & \text { cutoff wave number, all in } \\
& (1 / \mathrm{m}) ; \\
\lambda_{0}=(c / F) & \text { free space wavelength meters; } \\
\lambda_{\mathrm{g}} & \text { is the guided wavelength in me- } \\
& \text { ters; } \\
\lambda_{\mathrm{c}} & \text { is the cutoff wavelength in me- } \\
& \text { ters. }
\end{array}
$$

Guided and cutoff wavelengths are related by the well-known Ortusi relation

$$
\frac{1}{\lambda_{\mathrm{c}}^{2}}=\frac{1}{\lambda_{0}^{2}}-\frac{1}{\lambda_{\mathrm{g}}^{2}}
$$

Therefore, for a given frequency and waveguide mode, the propagation constant $\gamma$ has a prescribed value, depending on the mode order $(n, m)$ and the frequency.

$$
\gamma_{\mathrm{nm}}=\frac{2 \pi}{\lambda_{\mathrm{g}}}=\frac{2 \pi}{\lambda_{\mathrm{c}, \mathrm{nm}}} \sqrt{1-\left(\frac{f}{f_{\mathrm{c}, \mathrm{nm}}}\right)^{2}} .
$$

By inspection, it can be seen that at high frequencies $\gamma$ is imaginary, and the $z$ dependence form $e^{-\gamma z}$ gives a field varying sinusoidally along the guide.

At low frequencies, $\gamma$ is real and the field amplitude decays exponentially along the guide. So, little energy gets through due to these evanescent modes occurring below the cutoff frequencies.

Hence, for operating frequency $f$, much below the cutoff frequency $f_{\mathrm{c}, \mathrm{nm}}$, the attenuation constant is given by

$$
\gamma_{\mathrm{nm}}=\alpha_{\mathrm{nm}} \approx \frac{2 \pi}{\lambda_{\mathrm{c}, \mathrm{nm}}} .
$$

In a circular cylindrical waveguide of radius $a$, the cutoff wavelength is given by

$$
\begin{aligned}
\lambda_{\mathrm{c}, \mathrm{nm}} & =\frac{2 \pi a}{p_{\mathrm{nm}}^{\prime}}\left(\mathrm{TE}_{\mathrm{nm}} \text { or } H_{\mathrm{nm}} \text { modes }\right) \\
\lambda_{\mathrm{c}, \mathrm{nm}} & =\frac{2 \pi a}{p_{\mathrm{nm}}}\left(\mathrm{TM}_{\mathrm{nm}} \text { or } E_{\mathrm{nm}} \text { modes }\right)
\end{aligned}
$$

where $p_{\mathrm{nm}}$ and $p_{\mathrm{nm}}^{\prime}$ are roots of Bessel functions and their derivatives, respectively [8], [9].

Noting that higher order modes have higher cutoff frequencies, only the fundamental or dominant mode, having the lowest cutoff frequency, is of significance.

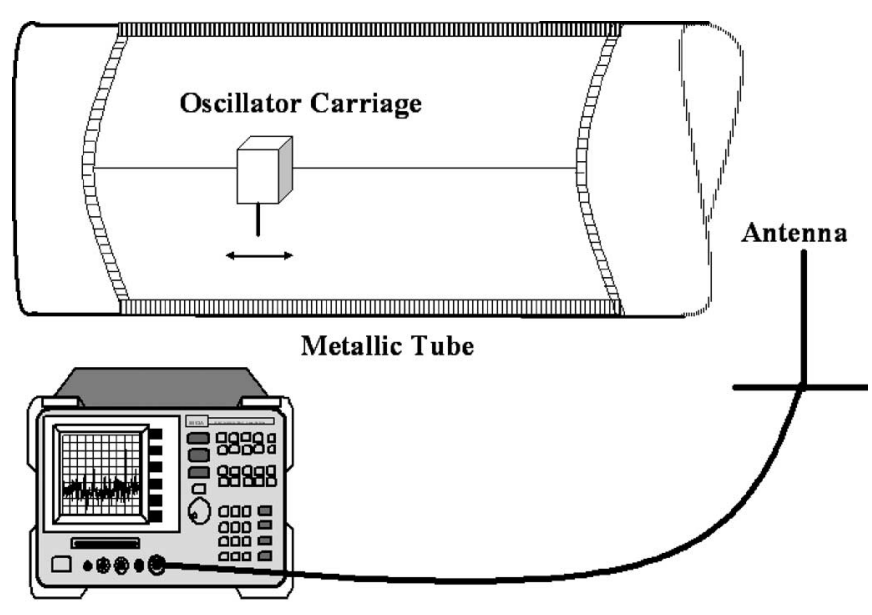

\section{SPECTRUM ANALYZER}

Fig. 2. Setup for shielding measurement.

Accordingly, for the cylindrical guide dominant mode $\mathrm{TE}_{11}$, attenuation is given by

$$
\alpha=\frac{2 \pi}{\lambda_{c, 11}}=\frac{1.841}{a}(\text { Neper } / m) .
$$

Therefore, a circular waveguide with diameter $D$ and length $L$ achieves, at frequencies below the cutoff, a shielding loss given by

$$
\alpha(d B)=8.686 \alpha L \cong 32 \frac{L}{D} .
$$

In practice, this means that for apertures acting as waveguides, the size should be selected such that it remains below the lowest cutoff frequency at the highest interference frequency.

\section{TEST EQUIPMENT}

To gain some insight into the relationship between the shielding loss and the characteristics of circular waveguides, the measurement setup shown in Fig. 2 is used to measure leakage of electromagnetic energy through a guide. This assembly comprises a circular waveguide with a diameter $D=21 \mathrm{~cm}$ and length $L=1.5 \mathrm{~m}$ (a ventilation duct or any metallic pipe could be used), a 50-MHz oscillator (built or ready to use), a dc power supply, 9-V battery, a dipole or loop antenna, and a spectrum analyzer.

Before performing experiments, an oscillator generating stable frequencies up to $2 \mathrm{GHz}$ with sufficient levels is realized.

The background theory of oscillators has been developed by many authors [11]-[13]. These references cover common theory of oscillators, provide practical design equations, and discuss requirements of the circuitry (active devices, resonators, etc.).

There are two types of transistor oscillators: oscillators constructed with amplifiers and their feedback circuit and others built with negative resistance one-ports.

For the formers, the concept is straightforward from the basic circuit and loop gain shown in Fig. 3. 


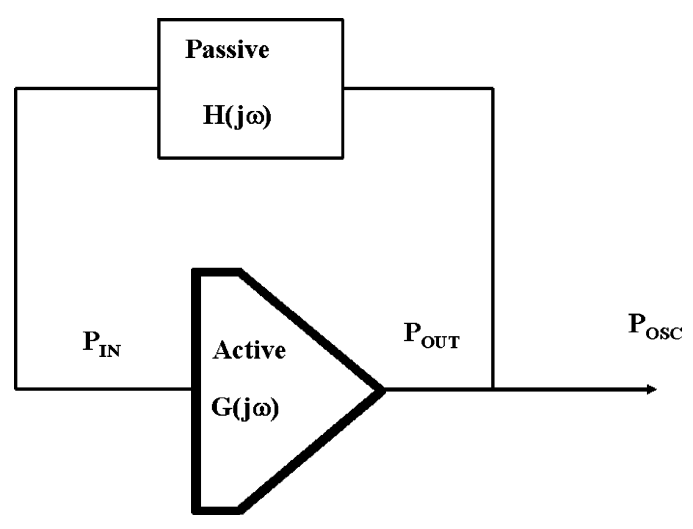

Fig. 3. Schematic of a feedback oscillator.

The total gain is unity and the total phase shift is forced to $360^{\circ}$ at the frequency of interest in order to have oscillations.

$$
G(j \omega) H(j \omega)=1 \Leftrightarrow\left\{\begin{array}{l}
|G(j \omega) H(j \omega)|=1 \\
\angle G(j \omega)+\angle H(j \omega)=0(2 \pi)
\end{array} .\right.
$$

Negative resistance oscillators design, on the other hand, proceeds as follows. First, a potentially unstable transistor is selected. The stability criterion is determined by the Rollet factor $K$, calculated from the small signal $S$-parameters [11]:

$$
K=\frac{1+|\Delta|^{2}-\left|S_{11}\right|^{2}-\left|S_{22}\right|^{2}}{2\left|S_{12}\right|\left|S_{21}\right|}
$$

where $\Delta=S_{11} S_{22}-S_{12} S_{21}$.

A transistor is unconditionally stable at any frequency when $K>1$ and $|\Delta|<1$. In this case, instability can be achieved by configuration changes (common emitter, common collector, and common base) or feedback addition (series or parallel reactive components).

Then, a resonator (LC, Quartz crystal, transmission lines, etc) is placed at one side of the unstable two-port to provide a negative resistance at the output port. The final step terminates this one-port with the appropriate load (generally $50 \Omega$ ).

RF CAD tools such as Advanced Design Systems of Agilent technologies are adequate to design the matching or feedback elements and to evaluate the performances of the oscillator (output power, noise, harmonics, etc.). This is achieved by design guides that guide engineers through multistep simulation and design procedure of complete devices such as oscillators.

Moreover, many ready-for-use oscillators built by manufacturers for different purposes are available at very low prices. These solid state oscillators that are built or bought present many advantages.

1) They are low cost and compact, making measurements in small enclosures possible.

2) They are powered by a battery, which is an efficient mean to prevent them from interferences traveling through power supply cables.

3) Combined with nonlinearities, they permit performance of measurements over several hundreds of megahertz, at once.
4) Test points or harmonics are well-identified integer multiples of the fundamental oscillation frequency. This implies more immunity to external ambient signals.

5) They can be loaded with monopole or loop antennas, creating electric or magnetic fields, as desired.

It is worthy to note that to use these oscillators at several hundreds of megahertz, a pair of parallel front-to-back diodes is placed at the output. Frequency multiplication or harmonic generation occurs due to this nonlinear combination, and the output signal is a harmonic series of the form [13], [14]

$$
S_{\text {out }}=S_{0}+S_{1} \cos \left(\omega t+\phi_{1}\right)+\ldots+S_{n} \cos \left(n \omega t+\phi_{n}\right) \text {. }
$$

In fact, this equation shows that we can get higher order frequencies multiples of the fundamental one and equidistant by a few megahertz.

The whole compact oscillator board is then mounted on a thin Plexiglas rod to slide easily along the guiding structure.

Care must be taken to avoid excessive rod dimensions with high permittivity since this may alter the field configuration and cause measurements errors.

A spectrum analyzer $(50 \Omega)$ suitable for sweeping the frequency range $50 \mathrm{MHz}$ to $1.5 \mathrm{GHz}$ and an antenna are used to measure the received signal emanating from the guide aperture. Outputs of the spectrum analyzer are $X-Y$ displays on a CRT screen. The horizontal axis is calibrated in frequency that increases linearly from left to right. This is done by setting either the center frequency and the frequency range (span) or both the start and stop frequencies.

The vertical axis is calibrated in amplitude in a linear scale (volts) or in a logarithmic scale (decibels). The log scale, which is often used, has a much wider usable range and allows measurements of either the absolute value of a signal or the amplitude difference between signals.

The calibration of the system is achieved by taking a reference level corresponding to any position of the oscillator in the vicinity of one end of the guide. The position of the oscillator carriage is then moved inside the structure and the spectrum analyzer readings are recorded at points located on a centimeter scale along the waveguide's axis.

This measurement setup matches the required specifications in that it does the following.

1) It uses available spectrum analyzers, solid state generators, and common antennas.

2) It covers the frequency range from a few Megahertz to several Gigahertz, namely, from the fundamental frequency of the oscillator to the highest harmonic level detectable by the spectrum analyzer or any other receiver.

3) It has a dynamic range equal to that of the spectrum analyzer and can be increased up to more than $120 \mathrm{~dB}$ by using an amplifier.

4) It can be extended from apertures characterization to check various enclosures effectiveness.

\section{DESIGN, EXPERIMENTS, AND RESULTS}

The first designed oscillator is a 55-MHz LC oscillator based on the $2 \mathrm{~N} 2219$ bipolar transistor. An 8-mm diameter air core 


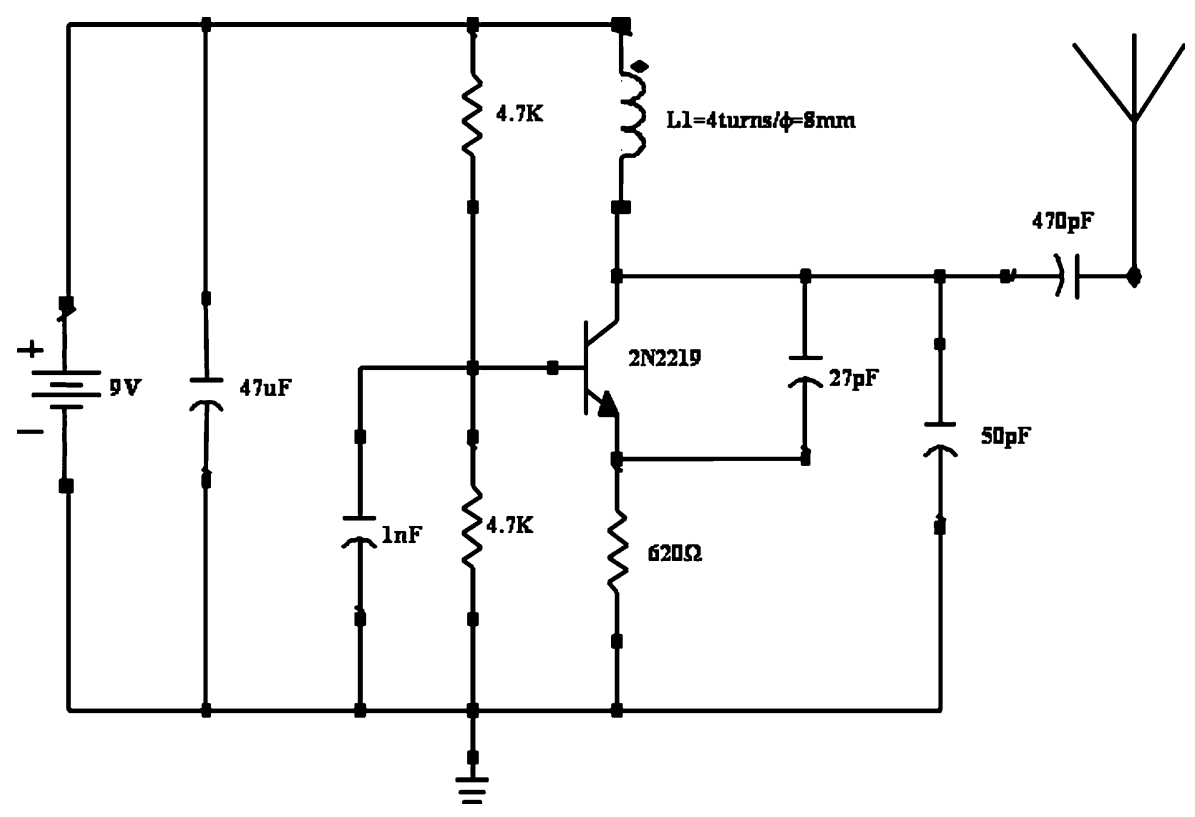

Fig. 4. Schematic of a transistor lumped oscillator.

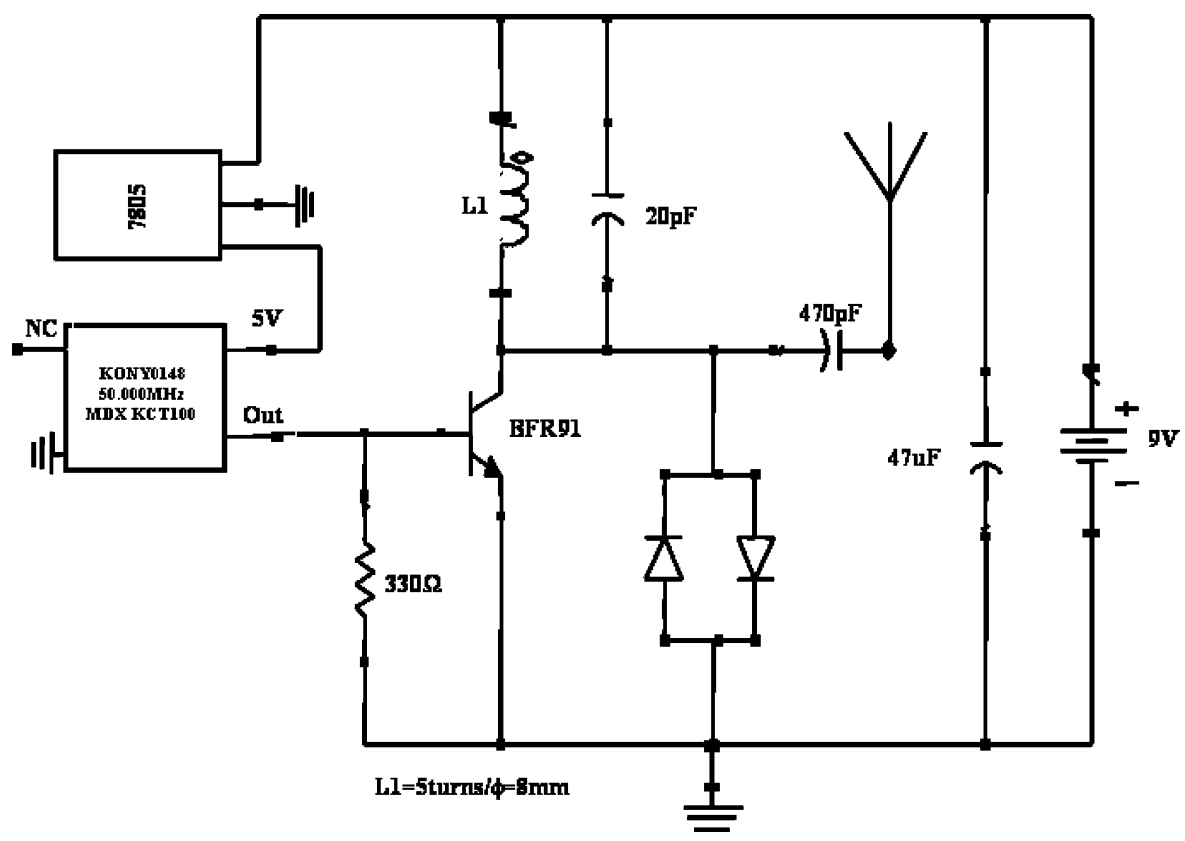

Fig. 5. Schematic of an integrated quartz oscillator.

inductance is used with conjunction of lumped capacitors to realize a lumped resonator, as shown in Fig. 4.

This oscillator exhibits excellent phase noise performance and its measured output power is about $3 \mathrm{dBm}$. However, care must be taken to enhance oscillations stability, which is seriously affected by discrete components tolerance and variations as well as vibration, load changes, power supply deviations, and closely placed conducting materials.

Another buffered oscillator (Fig. 5) based on a mounted surface package with a BFR91 amplifier has been built and tested. It showed significant advantage of quartz crystal. This oscillator delivers $-2-\mathrm{dBm}$ to $50-\Omega$ load. Its frequency of $50 \mathrm{MHz}$ is very stable over temperature, and its phase noise at $10 \mathrm{KHz}$ from the carrier is about $-98 \mathrm{dBc} / \mathrm{Hz}$ (Fig. 6).

As displayed in Fig. 7, harmonics up to $2 \mathrm{GHz}$ have sufficient amplitude levels to make wideband measurements using this $50-\mathrm{MHz}$ single tone generator.

The amplitude and frequency of every oscillator harmonic over the entire range are reported to check cutoff frequency as well as evanescent and propagating modes, as illustrated by Fig. 8. This is achieved by placing the carriage in the middle of the tube and reporting the emanating signals. The measured cut-off frequency of $850 \mathrm{MHz}$ is very close to the theoretical value of $837 \mathrm{MHz}$. 


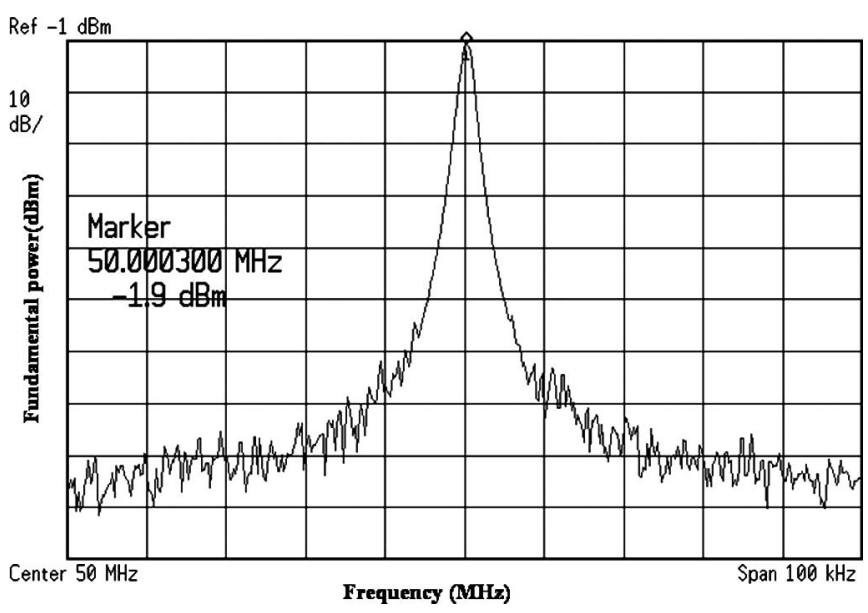

Fig. 6. Fundamental power and noise of a 50-MHz crystal oscillator.

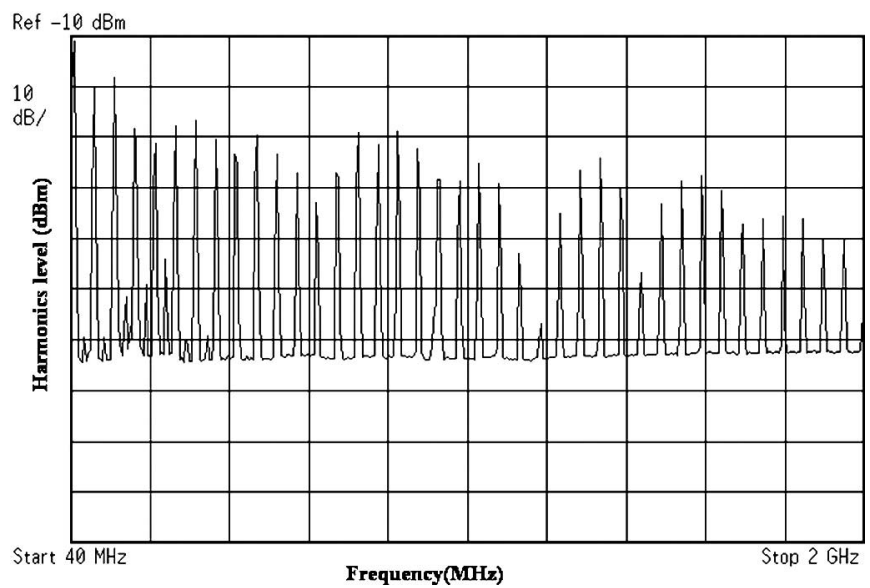

Fig. 7. Harmonics of a 50-MHz crystal oscillator with nonlinear termination.

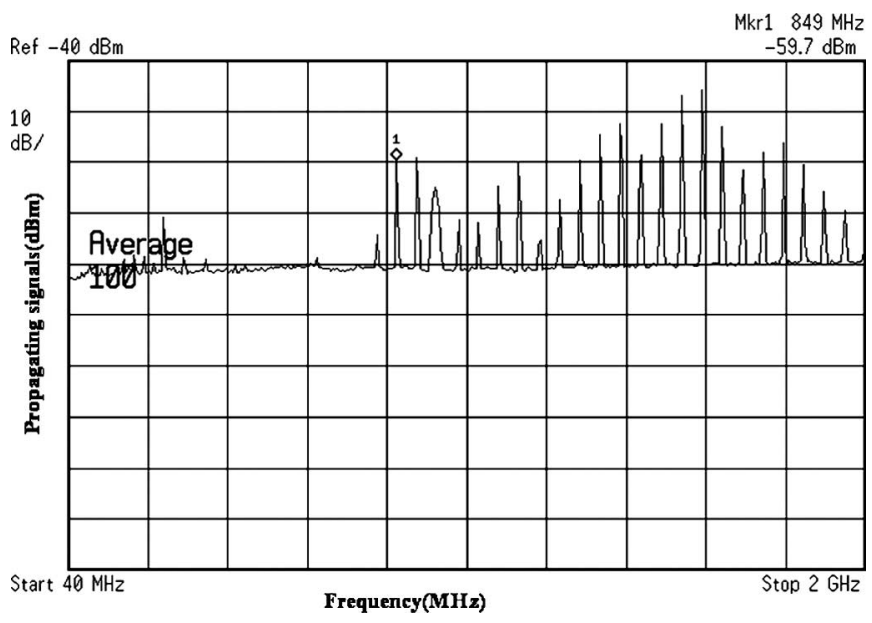

Fig. 8. Measured propagation and cutoff frequencies.

Then, the guide attenuation is plotted versus antenna position for several frequencies below cutoff. Some carriers of Radio, $\mathrm{TV}$, and GSM are within the desired spectrum, so care must be paid to avoid these ambient interferences when performing measurements (Fig. 9).

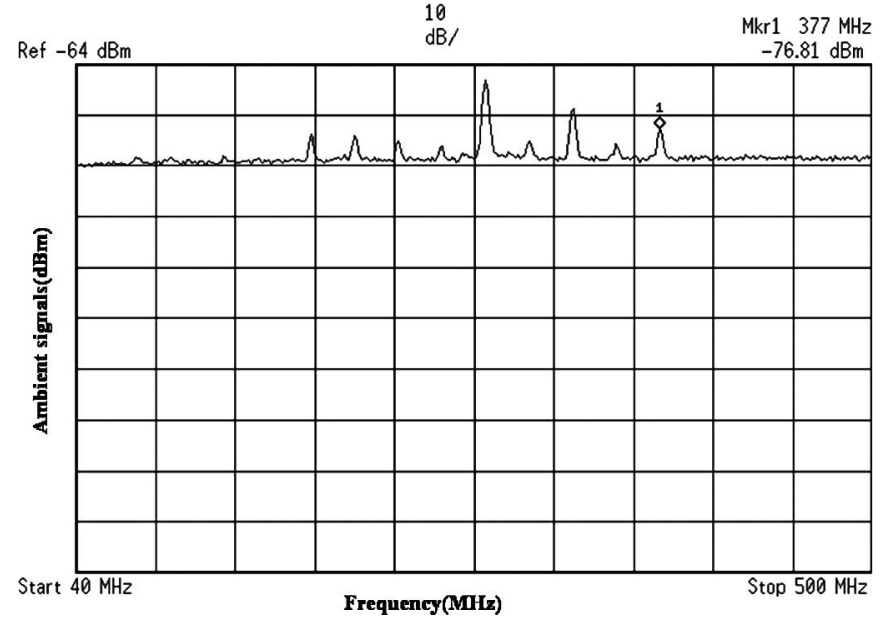

Fig. 9. Measured Ambient signals.

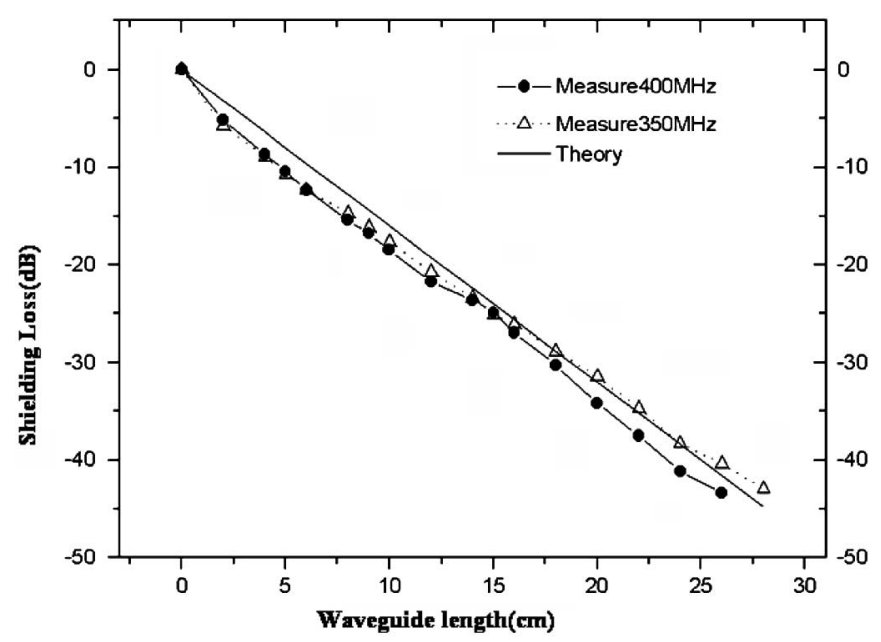

Fig. 10. Shielding loss in the waveguide below cutoff frequencies.

The definition of shielding is a relative concept dependent on the system configuration and general EMI protection goals.

Too many definitions of electromagnetic shielding and shielding effectiveness, which are often used, might include the following:

- ratios of incident and transmitted electric or magnetic fields through a specified shield, measured in free space or in a particular enclosure;

- electromagnetic loss in the shield;

- power reflection and absorption in the shield;

- transfer impedance, etc.

Each definition reflects a certain philosophy of understanding and evaluating the shielding mechanisms.

When dealing with apertures' effects, one could consider the EMI energy transfer from the shield powered with a specified signal to the free space environment.

Practical measurements were performed to evaluate the circular guide attenuation at 350 and $400 \mathrm{MHz}$. Fig. 10 depicts normalized measured and calculated losses versus guide length 
(or carriage position). Good agreement is observed between experimental data, and results are given by (7).

\section{CONCLUSION}

This experiment is a good illustration of shielding mechanisms provided by metallic tubes. To avoid radiation emission, power and signal cables can be shielded using such a shielding structure. Displays and vent panels (microwave ovens, honeycomb vents, ventilation apertures, etc.) can be also properly shielded with a fine metal wire mesh, where each cell is equivalent to a waveguide working below cutoff frequencies.

Our discussion concentrates on specialized knowledge and experimental tips required to apply well-established EMC principles toward practical implementation.

The implemented technique of shielding measurement matches technical, economical, and regulatory requirements.

First, the equipment used is low cost, available in most RF laboratories, and needs no special preparation or calibration.

Second, the use of an independent solid-state oscillator powered with a small battery permits to combat strong ambient signals and common mode couplings due to the presence of various cables.

Loading this relatively low-frequency oscillator with nonlinear components is a novel and efficient method to perform wideband measurements at specific frequencies spaced by a few Megahertz.

This convenient mean also permits us to avoid testing at frequencies close to those of ambient signals which, hopefully, are not necessarily multiples of the oscillation frequency.

This work can be extended to study the dependence of shielding performance on size, shape, and location of diverse discontinuities.

\section{REFERENCES}

[1] D. R. J. White, Shielding Design Methodology and Procedures. Gainesville, FL: Interference Control Technologies, 1986.

[2] V. P. Kodali, Engineering Electromagnetic Compatibility: Principles, Measurements, and Technologies. New York: IEEE Press, 1996.

[3] J. A. Stratton, Electromagnetic Theory. New York: McGraw-Hill, 1941.

[4] R. F. Harrington, Time Harmonic Electromagnetic Fields. New York: McGraw-Hill, 1961.

[5] B. Audone and M. Balma, "Shielding effectiveness of apertures in rectangular cavities," IEEE Trans. Electromagn. Compat., vol. 31, no. 1, pp. 102-106, Feb. 1989.

[6] M. Li, S. Radu, J. Nuebel, J. L. Drewniak, T. H. Hubing, and T. P. VanDoren, "Design of airflow aperture arrays in shielding enclosures," in Proc. IEEE Trans. EMC Symp., Denver, CO, vol. 2, Aug. 1998, pp. 1059-1063.

[7] F. Olyslager, E. Laermens, D. D. Zutter, S. Criel, R. D. Smedt, N. Lietaert, and A. D. Clercq, "Numerical and experimental study of the shielding effectiveness of a metallic enclosure,"IEEE Trans. Electromagn. Compat., vol. 41, no. 3, pp. 202-213, Aug. 1999.

[8] L. C. Andrews, Special Functions for Engineers and Applied Mathematics. New York: MacMillan, 1985

[9] M. Abramowitz and I. A. Stegun, Handbook of Mathematical Functions. New York: Dover, 1965.

[10] G. Gonzalez, Microwave Transistor Amplifiers: Analysis and Design. Englewood Cliffs, NJ: Prentice-Hall, 1997.

[11] R. S. Carson, High Frequency Amplifiers. New York: Wiley, 1975.

[12] G. D. Vendelin, Design of Amplifiers and Oscillators by the S-Parameters Method. New York: Wiley, 1982.
[13] I. Bahl and P. Bhartia, Microwave Solid State Circuit Design. New York: Wiley, 1988.

[14] S. A. Maas, Nonlinear Microwave and RF Circuits. Norwood, MA: Artech House, 2003.

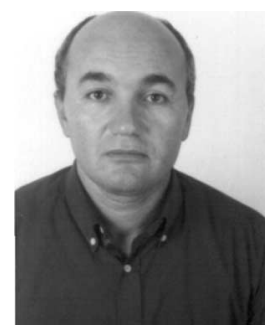

Fethi Choubani was born in Mahdia, Tunisia, in 1961. He received the electrical engineering diploma from Ecole National d'ingénieurs de Tunis, Tunis, Tunisia, in 1987 and the M.Eng. and Ph.D. degrees from ENSEEIHT, Institut National Polytechnique de Toulouse, Toulouse, France, in 1988 and 1993, respectively.

Since 1993, he has been teaching applied electronics and microwaves at Ecole Supérieure des Communications de Tunis (Sup'Com). He was manager of Telecommunications Department with Sup'Com from 1995 to 1996 and Director of strategy and prospection at Tunisie Télécom. His research interests are focused on oscillators and their applications in electromagnetic sensors. He is also interested in EMC, nonlinear modeling, and microwaves techniques.

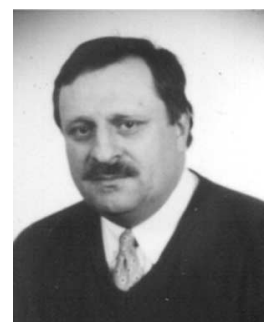

Jacques David (M'93) was born in France in 1946. He received the Ph.D. degree in electronicsmicrowaves from the University of Toulouse, Toulouse, France, in 1974 and the Docteur és Sciences degree in microwaves from the National Polytechnic Institute of Toulouse in 1984.

$\mathrm{He}$ is currently a professor with ENSEEIHT, Toulouse, where he has been teaching electromagnetics, microwave techniques, radar, instrumentation, and measurements since 1973. He has conducted several research projects, namely, in interaction wavesmatter, and dielectrics characterization.

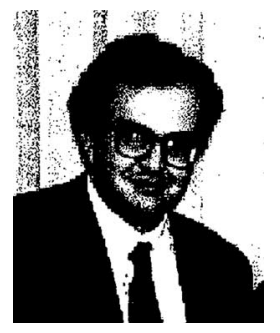

Nikos E. Mastorakis (M'97-SM'99) received the B.Sc. and M.Sc. (Diploma) degrees in electrical engineering from the National Technical University of Athens, Athens, Greece, in 1983 and 1988, respectively, and the $\mathrm{Ph} . \mathrm{D}$. degree in electrical engineering and computer science from the same university, in 1992. He also received the B.Sc. (Ptychion) degree in pure mathematics from the National University of Athens.

He served as special scientist on Computers and Electronics in the Hellenic (Greek) Army General Staff in 1993-1994 and taught several courses with the Electrical and Computer Engineering Department, National Technical University of Athens (1998-1994). $\mathrm{He}$ served as a Visiting Professor at the School of Engineering, University of Exeter, Exeter, U.K., in 1998 and as a Visiting Professor with the Technical University of Sofia, Sofia, Bulgaria, from 2003 to 2004). He has been a Full Professor and Head of the Department of Computer Science at the Military Institutions of University Education (MIUE), Hellenic Naval Academy, Greece, since 1994. 